\title{
Directional bonding explains the high conductance of atomic contacts in bec metals
}

\author{
W. Dednam $\odot,,^{1,2, *}$ C. Sabater $\odot,{ }^{2, \dagger}$ M. R. Calvo $\odot,{ }^{2}$ C. Untiedt $\odot,{ }^{2}$ J. J. Palacios $\odot,{ }^{3}$ A. E. Botha $\odot,{ }^{1}$ and M. J. Caturla $\odot^{2}$ \\ ${ }^{1}$ Department of Physics, Science Campus, University of South Africa, Private Bag X6, Florida Park 1710, South Africa \\ ${ }^{2}$ Departamento de Física Aplicada and Unidad Asociada CSIC, Universidad de Alicante, \\ Campus de San Vicente del Raspeig, E-03690 Alicante, Spain \\ ${ }^{3}$ Departamento de Física de la Materia Condensada, Condensed Matter Physics Center (IFIMAC), \\ and Instituto Nicolás Cabrera, Universidad Autónoma de Madrid, 28049 Madrid, Spain
}

(Received 12 August 2019; accepted 25 March 2020; published 20 April 2020)

\begin{abstract}
Atomic-sized contacts of iron, created in scanning tunneling microscope break junctions, present unusually high values of conductance compared to other metals. This result is counterintuitive since, at the nanoscale, body-centered-cubic metals are expected to exhibit lower coordination than face-centered-cubic metals. In this work we first perform classical molecular dynamics simulations of the contact rupture, using two different interatomic potentials. The first potential is isotropic, and produces mostly single-atom prerupture contacts. The second potential accounts for the directional bonding in the materials, and produces mostly highly coordinated prerupture structures, generally consisting of more than one atom in contact. To compare the two different types of structures with experiments, we use them as input to density functional theory electronic transport calculations of the conductance. We find that the highly coordinated structures, obtained from the anisotropic potential, yield higher conductances which are statistically in better agreement with those measured for body-centered-cubic iron. We thus conclude that the directional bonding plays an important role in body-centered-cubic metals.
\end{abstract}

DOI: 10.1103/PhysRevB.101.165417

\section{INTRODUCTION}

Stretching a metallic nanowire results in a progressive reduction of its cross section at the weakest point, until it finally breaks. From an atomistic viewpoint, when the minimum cross section of the nanowire contains only a few atoms, and for very slow stretching, the minimum cross section can in fact decrease by one atom at a time [1,2]. It seems reasonable to assume that the ultimate stable contact that holds the metal together is a single atom. Indeed, measurements of conductance for atomically sharp contacts seem to point in this direction since the stable contact conductance before rupture for most metals is just above one quantum, except for some notable exceptions; such as, iron [3,4], tantalum, molybdenum, and tungsten [5,6]. In fact, it is still widely assumed that the chemical valence of the bridging atom in single-atom contacts primarily determines the prerupture conductance values $[4,7,8]$.

Atomic-sized contacts are typically realized via mechanically controllable break junctions (MCBJs) $[9,10]$ or scanning tunneling microscope break junctions (STM-BJs) [11,12] and characterized through electron transport measurements. Classical molecular dynamics (CMD) simulations and firstprinciples transport calculations have been key in providing interpretations of the experimental results [1]. In the past decade, through the combination of experiments and simulations, researchers have identified different atomic contact geometries that may form just before rupture [13-16] and

\footnotetext{
*wd2@alu.ua.es

†carlos.sabater@ua.es
}

stated the important role played by the nearest-neighbor atoms in the mechanical and electrical properties of these structures [17].

Computationally, most of the systems studied so far have been face-centered-cubic (fcc) crystals, which exhibit a high probability to form single-atom contacts immediately before rupture. However, recent calculations [3] show a clear mismatch between experiment and theory in the case of bodycentered-cubic (bcc) iron. The reason behind this discrepancy is not clear, since three factors could play a role: chemical valence, crystal lattice structure, and/or magnetism.

To shed light on this question, we first perform CMD simulations of the iron rupture process, using two different interatomic potentials: one in which the bonding between atoms is treated as isotropic, the other in which the bonds also have covalent character. Density functional theory (DFT) calculations on CMD snapshots of the atomic configurations are then used to obtain the electronic transport properties (the conductance) and to compare with experimental data obtained from electron transport experiments in an STM-BJ at low temperature $(4.2 \mathrm{~K})$.

\section{EXPERIMENT}

In the STM-BJ configuration, the electrode tip can collide with and be withdrawn from the surface over continuous cycles of rupture and formation of the atomic-sized contact. The electrode tip consists of iron wire of nominal diameter $0.25 \mathrm{~mm}$ (with a purity of $99.99 \%$ ), and is connected in an electrical circuit as shown in Fig. 1(a), where a constant bias voltage $V=100 \mathrm{mV}$ is connected in series with the IV converter amplifier and, in turn, to a resistor of interest (in 
(a)

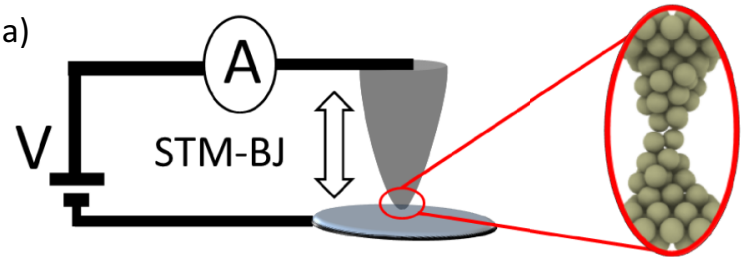

(b)

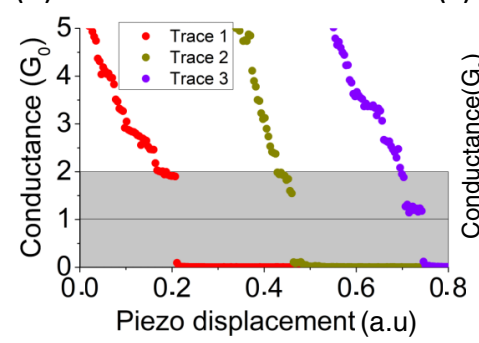

(c)

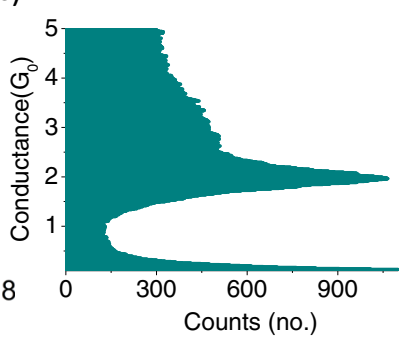

FIG. 1. (a) Experimental STM-BJ setup. (b) Traces of conductance. (c) Histogram of conductance constructed from more than 500 rupture traces of iron.

our case an atomic-sized contact). We express conductance that is the inverse of the resistance, in units of the quantum of conductance $G_{0}=2 e^{2} / h$, where $e$ is the charge of the electron, $h$ is the Planck constant, and the factor of 2 accounts for the spin degeneracy.

Typically, we record the conductance as a function of the relative displacement between the electrodes. The resulting curves are called rupture traces, as shown in Fig. 1(b). From each trace, we can build a histogram of conductance, which reveals the most frequent conductance values that the trace contains. Upon accumulating a significant number of individual histograms (one for every trace), we can construct a full histogram of conductance. For iron, it exhibits a clear peak at $\approx 2 G_{0}$ [see Fig. 1(c)], as has been previously observed $[3,4,18]$.

\section{THEORETICAL MODEL}

\section{A. Molecular dynamics simulations}

In CMD simulations the trajectory of each atom is obtained by solving Newton's second law for a semiempirically fitted interatomic potential. The hope is that the potential adequately describes the interactions between the atoms, right down to the atomic level [19]. In the case of metals, the problem of developing the most accurate interatomic potential has enjoyed considerable attention. Currently, the most extensively used potentials are those obtained from the embedded atom method (EAM) $[2,3,16,17,20,21]$. In the EAM the potential is assumed to be isotropic. While this assumption is valid for fcc structures, bcc structures have a lower coordination about an individual atom and thus exhibit more directionality in their bonding, i.e., slightly covalent character [22]. Accordingly, the modified embedded-atom method (MEAM) potential [23] may provide a more realistic description of the bonding in bcc metals, since directionality is included. In this work we thus compare the MEAM and EAM methods in order to determine which will lead to better agreement with experimental

(a)

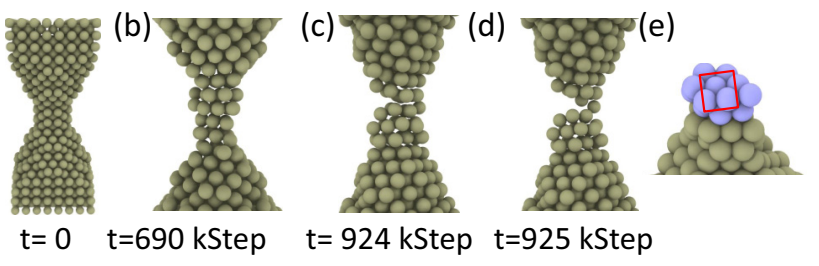

FIG. 2. (a) A typical initial input structure used in the simulations. Panels (b)-(d) show the process of rupture which occurs in 20 out of the 100 simulations of rupture with the MEAM potential [29]. The bcc iron contact goes through a crystallographic reorientation under tension, from having (001) to (110) planes perpendicular to the length of the contact [shown in (b)]. Rupture occurs in these cases via cleavage of (110)-oriented planes [shown in (e)]. The whole process lasts only a few picoseconds, e.g., from the structures in (c) and (d). (e) A cutaway (the top half of the contact has been removed) showing the characteristic five-atom structure (red rectangle) in a (110) surface of a bcc lattice.

results in electronic transport calculations on CMD-generated structures.

For both potentials, we use the large-scale atomic/ molecular massively parallel simulator (LAMMPS) [24,25]. Additionally, to imitate the experimental conditions, all the simulations are realized using a Nosé-Hoover thermostat $[26,27]$ to maintain a constant temperature. The thermostat is applied at the recommended interval of 1000 simulation time steps [25]. We use a time step of $1 \mathrm{fs}$ and the same initial input structure, consisting of $\approx 1500$ atoms, for comparison of the two potentials. Figure 2 shows a representative example of the rupture process of iron using the MEAM potential, with the atoms initially occupying positions in a perfect bcc lattice oriented along the (001) crystallographic direction [see Fig. 2(a)]. The initial velocities of the atoms are randomized at the beginning of each rupture run and correspond to an average temperature of $4.2 \mathrm{~K}$. The input structure is stretched at $\approx 1 \mathrm{~m} / \mathrm{s}$ until rupture. During every single rupture simulation, out of an ensemble of 100 independent runs performed with each potential, we compute the number of atoms in the minimum cross section of the model contact by means of the Bratkovksy algorithm [28]. The minimum cross section and simulation trajectory are both recorded every picosecond. For the purpose of comparison, traces are truncated $100 \mathrm{ps}$ before the moment of rupture when constructing cross-section histograms.

Figure 3 compares the two normalized histograms of minimum cross-section data obtained with each of the two potentials. The pink-shaded histogram in Fig. 3 has been constructed by using the most recent MEAM interatomic potential, fitted to the melting point of Fe as well as its nearmelting point elastic constants [29]. This particular potential is suitable for simulations of $\mathrm{Fe}$ contact rupture because the (001), (110), and (111) exposed surface energies agree very well with experiments [29]. For comparison, see the blueshaded minimum cross-section histogram in Fig. 3, obtained using an EAM potential whose surface energies also agree reasonably well with experiment and DFT calculations [20]. Although both potentials show the formation of one- and two-atom contacts (first two peaks in the histogram), the 


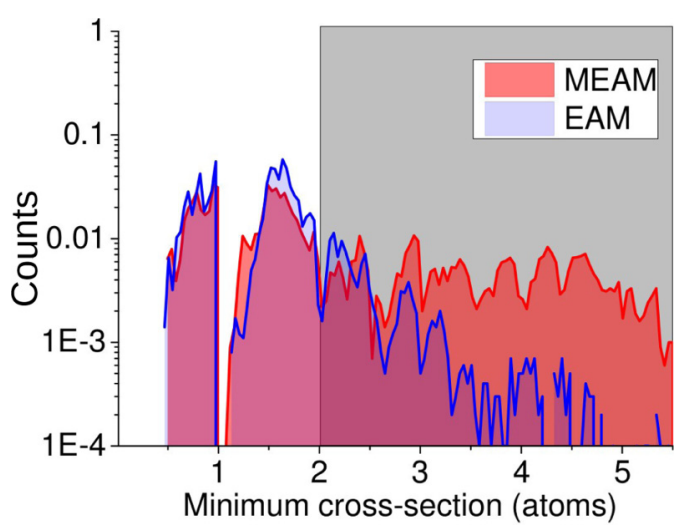

FIG. 3. Minimum cross-section histograms obtained after 100 rupture simulations using the MEAM potential [29] (pink shading with red outline) and the EAM potential [20] (grey shading with blue outline).

MEAM potential produces stable prerupture structures with a higher number of atoms in the minimum cross section than the EAM potential. This is shown in the shaded tail of the histogram, where the probability of forming structures with more than two atoms right before rupture is lower with the EAM potential than with MEAM.

Note that in the case of fcc metals [13-15,17], and even in the structures obtained with the EAM potential for Fe, the simulated cross section narrows atom by atom during the rupture process. In contrast, the cross section obtained using the MEAM potential breaks through cleavage across (110) oriented planes as shown in Fig. 2(e). These (110) planes are formed after a reorientation of the contact during the applied tension.

\section{B. Density functional theory quantum transport calculations}

To obtain the conductance of snapshots extracted from CMD simulations, such as in Fig. 2(c), we use the electronic transport code Alicante nanotransport (ANT . Gaussian) [30-34]. (For more details of the DFT calculations, see the Appendix.) To improve the quality of conductance results, an all-electron basis set has been assigned to 15-20 atoms in the minimum cross section of the trimmed-down inputs for conductance calculations. The all-electron basis set was optimized in CRYSTAL14 [35] after adding uncontracted Gaussian-type orbitals to an existing basis set for $\mathrm{Fe}$, and varying their coefficients and exponents in the same way as was done for Ni in Ref. [36]. The quality of the basis set has been verified by comparing the band structure it produces for bulk bcc Fe with that produced by OpenMX [37]. Conductance values near the peak of the experimental histogram in Fig. 1(c) are obtained for structures which correspond to those shown in Fig. 2(c) [see cases marked with an asterisk (*) in Table I of the Appendix], that is, those predicted by the MEAM potential. Note that the EAM potential only reproduces the rupture process illustrated in Fig. 2 in three out of the 100 rupture simulations versus 20 out of 100 in the case of the MEAM potential.

More revealing than the statistical study presented above are the so-called Fano factors [21]. In experiments at low tem- (a)

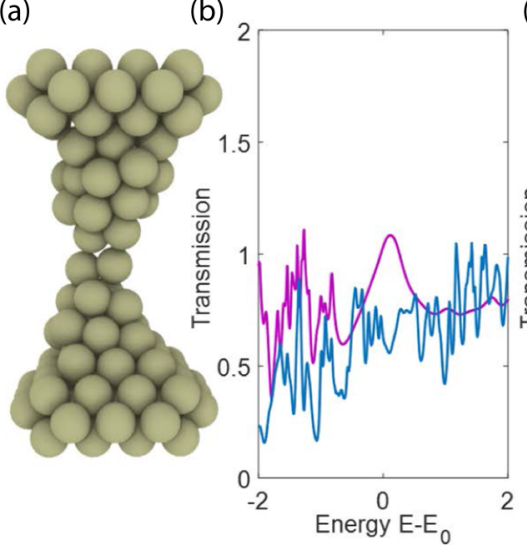

(c)

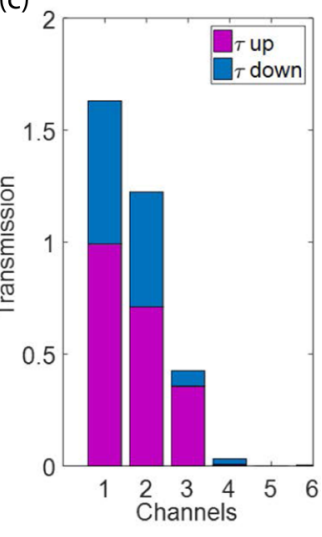

FIG. 4. (a) Fe double contact, (b) overall spin-resolved transmission vs energy (eV), (c) transmission vs spin eigenchannel. A Fano factor of 0.3084 is obtained from the eigenchannels shown in (c).

peratures, low bias voltage, and low frequency range (maximum of $400 \mathrm{kHz}$ ), the Fano factor $F$ provides a measure of noise suppression relative to the maximum Poissonian value of $2 e I$ [21]. Therefore, the shot noise from ballistic transport of an electron through an atomic-sized contact is given by $S_{I}=2 e I F$, where $I$ is the bias current and $e$ is the electron charge. On the other hand, in DFT quantum transport calculations, the spin-polarized conductance can be expressed as [21] $G=\frac{G_{0}}{2} \Sigma_{n, \sigma} T_{n, \sigma}$, where $G_{0}$ is the usual spin-degenerate quantum of conductance and $T_{n, \sigma}$ are the individual spinresolved eigenchannel transmissions [38]. Since not only the geometry but also the number of atoms in the constriction of a contact determines the overall conductance through their valence orbitals, the individual spin-resolved transmission channels can convey information about the atomic structure of the contacts through the Fano factor (a measure of the number of partially open transmission channels in an atomic-sized contact):

$$
F=\frac{\Sigma_{n, \sigma} T_{n, \sigma}\left(1-T_{n, \sigma}\right)}{\Sigma_{n, \sigma} T_{n, \sigma}} .
$$

Figure 4 shows an example of how an eigenchannel analysis can be carried out to obtain the Fano factor from a conductance calculation on a model contact. A conductance calculation on the structure shown in Fig. 4(a) not only yields the overall spin-resolved transmissions in Fig. 4(b) (which sum to $G=1.6574 G_{0}$ ), but also the contributing spin-resolved eigenchannel transmissions, shown in Fig. 4(c). Therefore, in this case only five spin-resolved eigenchannels - three spinmajority (purple) and two spin-minority (blue)—contribute significantly to the overall transmission. Based on this analysis, one can conclude that at least two atoms effectively contribute to the transmission in this contact. The noise in the transmission functions in Fig. 4(b) result from the disorder in CMD structures in general [39] and, in particular, from the spd hybridization of the spin-minority channels [40].

\section{RESULTS AND DISCUSSION}

Fano factors calculated from CMD snapshots of the two CMD potentials are presented in Fig. 5. The dark grey areas 


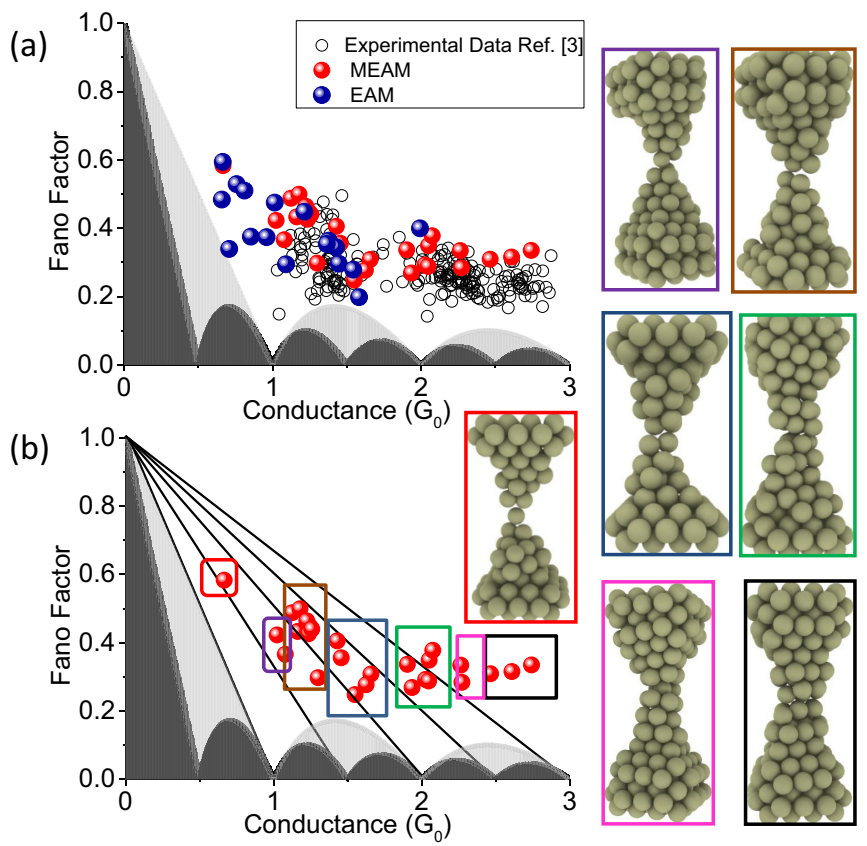

FIG. 5. (a) Fano factors vs conductance for structures obtained via the EAM (blue markers) and MEAM (red markers) potentials. Also shown in (a) are the experimental data points from Ref. [3] (reproduced here with permission). The shaded regions delineate forbidden values of the Fano factors where light grey corresponds to spin-degenerate transmission channels and dark grey to spinresolved channels. (b) In the case of the MEAM potential, the data are grouped by color-coded frames according to stable structures just before rupture, shown in the insets. The conductance values are recorded in Tables I and III. The black diagonal lines determine the number of spin-resolved transmission channels, with one electron per channel, while light grey refers to spin-degenerate transmission channels. The agreement with the experimental values from Ref. [3] is quite remarkable.

delineate forbidden values of $F$ for magnetic atomic-sized contacts, while the areas underneath the light grey line are the forbidden values of $F$ for nonmagnetic materials. Recall that $F$ is a measure of the number of partially open transmission channels in a contact, and the more channels contribute to the overall conductance, the more atoms are likely involved.

For the MEAM potential, the calculated conductance values [plotted separately in Fig. 5(b)] fall at or near the four, five, and six transmission channel lines. Experimentally, $\mathrm{Fe}$ has been shown to form last-contact structures with six transmission channels [the experimental data from Fig. 6(b) of Ref. [3] are reproduced with permission in Fig. 5(a)], which appears to indicate the formation of contacts with three or more atoms in them, according to the values of the Bratkovksy minimum cross sections in Table I of the Appendix. The MEAM potential, with more covalent character, is thus seen to outperform the EAM potential in this regard, whose $F$ values for the 17 conductance values collected in Table III are also plotted in Fig. 5(a).

Moreover, based on the low density of states of $\mathrm{Fe}$ at the Fermi level [40], in comparison to $\mathrm{Ni}$ or $\mathrm{Co}$, one would expect $\mathrm{Fe}$ to have a first maximum conductance peak at a lower conductance value than either of the latter metals. The experimental Fano data from Ref. [3] shown in Fig. 5(a) exhibit a significant number of conductance values at this expected low value of $\approx 1.2-1.4 G_{0}$, but in a histogram, they are subsumed by the broad peak at $\approx 2 G_{0}$ (see fits to the histogram of conductance in the Appendix). Our interpretation, based on the simulation results presented above, is that slight differences in the structures responsible for this peak (see Fig. 2) could result also in deviations from the $2 G_{0}$ value. For similar reasons, related to the detailed structure of the contacts, magnetism is expected to play almost no role in iron in the absence of an external field, since it does not even in the case of Ni [41].

Therefore, we postulate that the discrepancy between the experiments and the simulations in the work of Vardimon et al. [3] for the case of Fe is the lack of CMD structures with minimum cross sections above $\approx 3$ atoms at rupture when using an EAM potential, in a combination with a tight-binding model to calculate conductance. Using a more detailed model of the bonding between $\mathrm{Fe}$ atoms, such as the MEAM potential used in this work, should markedly improve the comparison between the experimental histogram of conductance and the one calculated in [3] based on the good agreement between the theoretical Fano diagram in Fig. 5(b) and their experimental Fano diagram in Fig. 6(b) of [3].

It is also important to note that other bec materials such as $\mathrm{Ta}, \mathrm{Mo}$, and $\mathrm{W}$, in similarity with iron, show experimental histograms of conductance with pronounced peaks at around $\approx 2 G_{0}[5,6]$, and that the formation of similar structures could be explored in future work.

\section{CONCLUSION}

In summary, the pronounced peaks at $\approx 2 G_{0}$ that appear in experimental histograms of conductance of body-centeredcubic metal atomic contacts could not previously be explained by considering single-atom contact structures [29]. Here, we show that DFT electronic transport calculations of structures with several atoms in the minimum cross section give conductance values in agreement with experiments. Such structures arise when an energetically favorable reorientation occurs, from (001) to stable (011) layers perpendicular to the direction of stretching. We obtain this stable reorientation when using an interatomic potential (the MEAM potential) that includes directional bonding. Our findings contradict the presently held assumption that the most likely stable prerupture contacts in bcc metals are made up of a single atom. The MEAM potential thus provides a realistic mechanism of atomic rupture for $\mathrm{Fe}$ in which covalent bonding plays a key role. Body-centered-cubic metals such as $\mathrm{Fe}$ may therefore represent good candidates for producing molecular junctions in which the electrodemolecule interface is an atomically flat surface.

\section{ACKNOWLEDGMENTS}

We gratefully acknowledge the generosity of Prof. O. Tal and Dr. A. Vilan of the Weizmann Institute for sharing experimental data from Ref. [3] with us. This work was supported by the Generalitat Valenciana through PROMETEO2017/139 and GENT (CDEIGENT2018/028), the Spanish government through Grants No. MAT2016-78625-C2-1-P and No. 
FIS2016-80434-P, and the Spanish Ministry of Science and Innovation, through the "María de Maeztu" Programme for Units of Excellence in R\&D (CEX2018-000805-M), by Comunidad Autónoma de Madrid through Grant No. S2018/NMT-4321 (NanomagCOST-CM), by the Fundación Ramón Areces, and by the European Union Graphene Flagship under Grant No. 604391. J.J.P. acknowledges the computer resources and assistance provided by the Centro de Computación Científica of the Universidad Autónoma de Madrid and the RES. W.D. thanks M. Á. Fernández for useful discussions about the MEAM potential. The MD and DFT calculations in this paper were performed on the highperformance computing (HPC) facility at UNISA.

\section{APPENDIX}

This Appendix provides further details about the Gaussian fitting of the experimental histogram, as well as the DFT calculations described in the main paper. It also gives the results of conductance calculations on snapshots from CMD simulations performed with the MEAM and EAM potentials.

\section{Gaussian fitting functions}

In Fig. 6 we show how a sum of three Gaussian functions can be fitted to the experimental histogram in Fig. 1(c) of the main paper.

The blue markers represent the experimental data, and the red line through them is the sum of the three Gaussian curves, which are centered on $1.60,1.99$, and $2.39 G_{0}$, respectively. The fact that we need more than two Gaussian curves in order to fit the main peak of the experimental data implies that there is not a single, repeatable, stable structure giving rise to the experimentally observed peak. It implies that the peak is produced by a variety of different structures. On the other hand, the three centers of the Gaussians cover the range of calculated conductance values, shown in Table I, rather well.

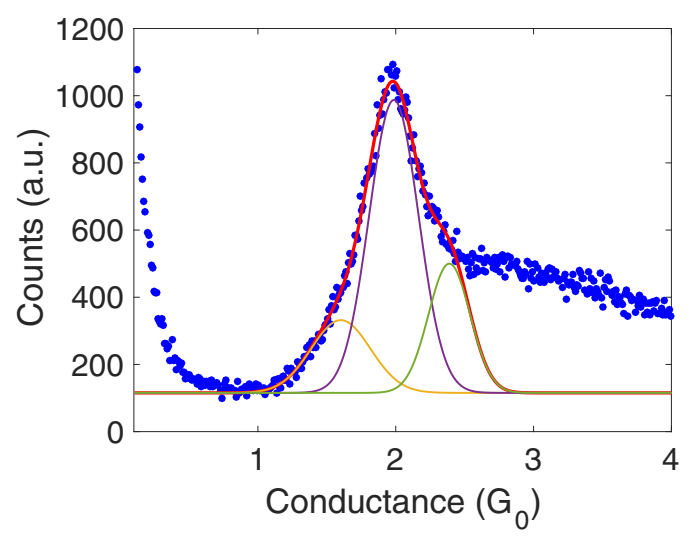

FIG. 6. A sum of three Gaussian functions fitted to the experimental conductance histogram obtained from the rupture of iron at $4.2 \mathrm{~K}$. Blue markers are the raw experimental data, while the yellow, purple, and green curves are Gaussian functions that sum to give the red line.
TABLE I. Contact type, Bratkvosky minimum cross section, and conductance of snapshots from CMD simulations with MEAM potential for Fe.

\begin{tabular}{|c|c|c|c|}
\hline Rupture & Type & Min. cross section & Conductance $\left(G_{0}\right)$ \\
\hline 2 & $5-3-4$ & 1.6 & 1.2 \\
\hline 5 & $9-7-8 *$ & 4.7 & 2.4 \\
\hline 8 & $8-6-9 *$ & 3.4 & 2.3 \\
\hline 11 & $2-1-2$ & 0.6 & 1.0 \\
\hline 12 & $6-6-7 *$ & 4.7 & 2.1 \\
\hline 15 & $5-3-5$ & 1.5 & 1.4 \\
\hline 17 & $9-6-8^{*}$ & 3.8 & 2.1 \\
\hline 18 & $5-2-2-5$ & 1.7 & 1.4 \\
\hline 19 & $7-4-8 *$ & 3.2 & 1.9 \\
\hline 34 & $3-2-2-2$ & 0.8 & 1.1 \\
\hline 40 & $8-6-9 *$ & 4.3 & 2.1 \\
\hline 46 & $4-2-4$ & 1.5 & 1.5 \\
\hline 49 & $3-2-5$ & 1.6 & 1.2 \\
\hline 51 & $4-2-4$ & 1.6 & 1.3 \\
\hline 54 & $6-3-6^{*}$ & 3.0 & 2.3 \\
\hline 55 & $4-2-2-5$ & 1.2 & 1.3 \\
\hline 56 & $5-2-4$ & 1.5 & 1.3 \\
\hline 58 & $3-2-4$ & 1.4 & 1.1 \\
\hline 60 & $3-2-4$ & 1.6 & 1.6 \\
\hline 63 & $4-2-2-5$ & 1.4 & 1.3 \\
\hline 64 & $5-2-2-5$ & 1.7 & 1.9 \\
\hline 67 & $5-3-3-5$ & 1.3 & 1.3 \\
\hline 70 & $3-1-3$ & 0.8 & 1.2 \\
\hline 72 & $5-2-3$ & 0.8 & 1.5 \\
\hline 73 & $5-2-3$ & 1.4 & 1.1 \\
\hline 74 & $3-1-3$ & 0.9 & 1.0 \\
\hline 75 & $5-2-2-5$ & 1.6 & 2.0 \\
\hline 77 & $8-7-8 *$ & 4.0 & 2.3 \\
\hline 85 & $8-4-5^{*}$ & 2.8 & 1.9 \\
\hline 88 & $5-3-3-5$ & 1.5 & 1.6 \\
\hline 91 & $7-6-10 *$ & 4.2 & 2.5 \\
\hline 97 & 2-1-2 & 1.0 & 1.2 \\
\hline 99 & $4-2-3$ & 1.2 & 1.7 \\
\hline
\end{tabular}

The red line is expressed as

$$
y(x)=\Phi+\sum_{i=1}^{3} a_{i} e^{\left(x-b_{i}\right)^{2} / 2 c_{i}^{2}}
$$

where the coefficients $a_{i}$ are the amplitudes of the three underlying curve peaks, $b_{i}$ are the positions of their centers, and $c_{i}$ are the standard deviations. The constant $\Phi$ is an offset with a value of 104 counts for this fit. Table II collects all the fitting parameters.

TABLE II. Fitting parameters.

\begin{tabular}{lccc}
\hline \hline Gaussian & $a$ & $b$ & $c$ \\
\hline 1 & 216 & 1.60 & 0.30 \\
2 & 872 & 1.99 & 0.25 \\
3 & 385 & 2.39 & 0.21 \\
\hline \hline
\end{tabular}


TABLE III. Contact type, Bratkvosky minimum cross section, and conductance of snapshots from CMD simulations with EAM potential for Fe.

\begin{tabular}{lccc}
\hline \hline Rupture & Type & Min. cross section & Conductance $\left(G_{0}\right)$ \\
\hline 1 & $4-3-4$ & 2.5 & 1.2 \\
8 & $5-2-5$ & 1.6 & 1.6 \\
10 & $3-2-2-3$ & 1.5 & 1.0 \\
13 & $5-3-3-5$ & 1.7 & 1.4 \\
16 & $4-2-3$ & 1.7 & 1.4 \\
21 & $3-2-3$ & 1.5 & 1.4 \\
23 & $3-2-3$ & 1.7 & 1.5 \\
24 & $4-3-6$ & 1.6 & 2.0 \\
42 & $4-1-3$ & 0.8 & 0.9 \\
53 & $2-1-3$ & 0.7 & 1.0 \\
69 & $3-1-2$ & 0.7 & 0.8 \\
74 & $2-1-2$ & 0.7 & 0.7 \\
77 & $3-1-2$ & 0.7 & 0.8 \\
80 & $2-1-2$ & 0.8 & 1.1 \\
87 & $2-1-2$ & 0.6 & 0.7 \\
94 & $4-2-3$ & 1.3 & 1.4 \\
98 & $2-1-2$ & 0.5 & 0.7 \\
\hline \hline
\end{tabular}

\section{DFT calculations}

We extracted 33 representative snapshots of stable prerupture structures from the $100 \mathrm{CMD}$ simulations with the MEAM potential, ten of which correspond to the rupture process illustrated in Fig. 2(c) of the main paper. The snapshots were trimmed down to $\sim 200$ atoms centered on the minimum cross section to allow conductance calculations to finish in a reasonable time. The results of the conductance calculations

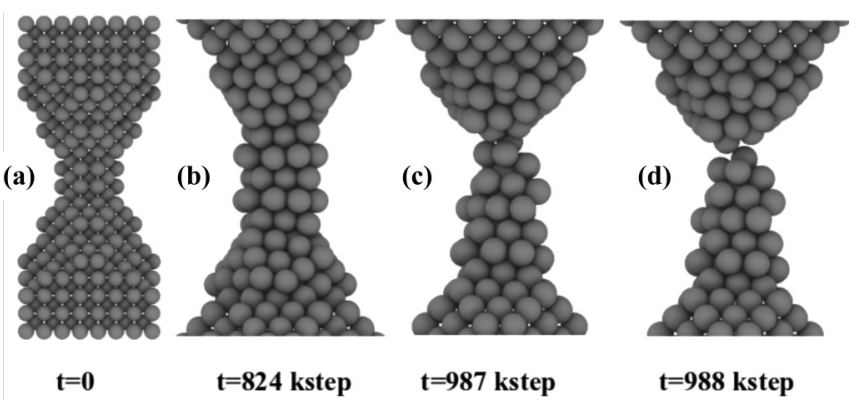

FIG. 7. (a) The same initial input structure as in Fig. 2(a) of the main paper, used in the simulations. Panels (b)-(d) show the process of rupture corresponding to rupture number 24 in Table III, obtained with the EAM potential [20]. The bcc iron contact goes through a crystallographic reorientation under tension, from having (001) to (110) planes perpendicular to the length of the contact [shown in (b)]. Rupture occurs in these cases via thinning to elongated wires, with at most three atoms in the minimum cross section several picoseconds before rupture.

are shown in Table I. On the other hand, 17 snapshots have been extracted from the $100 \mathrm{CMD}$ rupture runs performed with the EAM potential, which were also trimmed down for conductance calculations. The results of these calculations are collected in Table III. In addition, in Fig. 7 we show the prerupture structure obtained with the EAM potential which leads to the highest conductance, for rupture 24 , in Table III. The rupture mechanism is completely different than that shown in Fig. 2 of the main paper, obtained with the MEAM potential. The nanocontact undergoes thinning to elongated structures which are seen for other metals described by EAM potentials too [15].
[1] U. Landman, W. D. Luedtke, N. A. Burnham, and R. J. Colton, Atomistic mechanisms and dynamics of adhesion, nanoindentation, and fracture, Science 248, 454 (1990).

[2] N. Agraït, A. L. Yeyati, and J. M Van Ruitenbeek, Quantum properties of atomic-sized conductors, Phys. Rep. 377, 81 (2003).

[3] R. Vardimon, M. Matt, P. Nielaba, J. C. Cuevas, and O. Tal, Orbital origin of the electrical conduction in ferromagnetic atomic-size contacts: Insights from shot noise measurements and theoretical simulations, Phys. Rev. B 93, 085439 (2016).

[4] M. R. Calvo, J. Fernández-Rossier, J. J. Palacios, D. Jacob, D. Natelson, and C. Untiedt, The Kondo effect in ferromagnetic atomic contacts, Nature (London) 458, 1150 (2009).

[5] D. den Boer, O. I. Shklyarevskii, and S. Speller, Bias dependence of W, Mo and Ta conductance histograms, Phys. B (Amsterdam, Neth.) 395, 20 (2007).

[6] A. Halbritter, Sz. Csonka, G. Mihály, E. Jurdik, O. Yu. Kolesynchenko, O. I. Shklyarevskii, S. Speller, and H. van Kempen, Transition from tunneling to direct contact in tungsten nanojunctions, Phys. Rev. B 68, 035417 (2003).
[7] R. Requist, P. P. Baruselli, A. Smogunov, M. Fabrizio, S. Modesti, and E. Tosatti, Metallic, magnetic and molecular nanocontacts, Nat. Nanotechnol. 11, 499 (2016).

[8] E. Scheer, N. Agraït, J. C. Cuevas, A. L. Yeyati, B. Ludoph, A. Martín-Rodero, G. R. Bollinger, J. M. van Ruitenbeek, and C. Urbina, The signature of chemical valence in the electrical conduction through a single-atom contact, Nature (London) 394, 154 (1998).

[9] C. J. Muller, J. M. van Ruitenbeek, and L. J. de Jongh, Experimental observation of the transition from weak link to tunnel junction, Physica C 191, 485 (1992).

[10] C. J. Muller, J. M. van Ruitenbeek, and L. J. de Jongh, Conductance and Supercurrent Discontinuities in Atomic-Scale Metallic Constrictions of Variable Width, Phys. Rev. Lett. 69, 140 (1992).

[11] J. I. Pascual, J. Méndez, J. Gómez-Herrero, A. M. Baró, N. García, and V. T. Binh, Quantum Contact in Gold Nanostructures by Scanning Tunneling Microscopy, Phys. Rev. Lett. 71, 1852 (1993).

[12] N. Agraït, J. G. Rodrigo, and S. Vieira, Conductance steps and quantization in atomic-size contacts, Phys. Rev. B 47, 12345 (1993). 
[13] A. Hasmy, A. J. Pérez-Jiménez, J. J. Palacios, P. GarcíaMochales, J. L. Costa-Krämer, M. Díaz, E. Medina, and P. A. Serena, Ballistic resistivity in aluminum nanocontacts, Phys. Rev. B 72, 245405 (2005).

[14] A. Hasmy, E. Medina, and P. A. Serena, From Favorable Atomic Configurations to Supershell Structures: A New Interpretation of Conductance Histograms, Phys. Rev. Lett. 86, 5574 (2001).

[15] P. García-Mochales, S. Peláez, P. A. Serena, E. Medina, and A. Hasmy, Breaking processes in nickel nanocontacts: a statistical description, Appl. Phys. A: Mater. Sci. Process. 81, 1545 (2005).

[16] C. Sabater, M. J. Caturla, J. J. Palacios, and C. Untiedt, Understanding the structure of the first atomic contact in gold, Nanoscale Res. Lett. 8, 257 (2013).

[17] C. Sabater, W. Dednam, M. R. Calvo, M. A. Fernández, C. Untiedt, and M. J. Caturla, Role of first-neighbor geometry in the electronic and mechanical properties of atomic contacts, Phys. Rev. B 97, 075418 (2018).

[18] C. Untiedt, D. M. T. Dekker, D. Djukic, and J. M. van Ruitenbeek, Absence of magnetically induced fractional quantization in atomic contacts, Phys. Rev. B 69, 081401(R) (2004).

[19] D. C. Rapaport, The Art of Molecular Dynamics Simulation, 2nd ed. (Cambridge University Press, New York, 2004).

[20] L. Malerba, M. C. Marinica, N. Anento, C. Björkas, H. Nguyen, C. Domain, F. Djurabekova, P. Olsson, K. Nordlund, A. Serra, D. Terentyev, F. Willaime, and C. S. Becquart, Comparison of empirical interatomic potentials for iron applied to radiation damage studies, J. Nucl. Mater. 406, 19 (2010).

[21] J. C. Cuevas and E. Scheer, Molecular Electronics (World Scientific, Singapore, 2010).

[22] D. G. Pettifor, Bonding and Structure of Molecules and Solids (Oxford University Press, Oxford, 1996).

[23] M. I. Baskes, Modified embedded-atom potentials for cubic materials and impurities, Phys. Rev. B 46, 2727 (1992).

[24] S. Plimpton, Fast parallel algorithms for short-range molecular dynamics, J. Comp. Phys. 117, 1 (1995).

[25] S. Plimpton, A. Thompson, S. Moore, A. Kohlmeyer, and R. Berger, Computer code LAMMPS, 2016, publicly available at http://lammps.sandia.gov.

[26] S. Nosé, A molecular dynamics method for simulations in the canonical ensemble, Mol. Phys. 52, 255 (1984).
[27] W. G. Hoover, Canonical dynamics: Equilibrium phase-space distributions, Phys. Rev. A 31, 1695 (1985).

[28] A. M. Bratkovsky, A. P. Sutton, and T. N. Todorov, Conditions for conductance quantization in realistic models of atomic-scale metallic contacts, Phys. Rev. B 52, 5036 (1995).

[29] S. A. Etesami and E. Asadi, Molecular dynamics for near melting temperatures simulations of metals using modified embedded-atom method, Phys. Chem. Solids 112, 61 (2018).

[30] J. J. Palacios, A. J. Pérez-Jiménez, E. Louis, and J. A. Vergés, Fullerene-based molecular nanobridges: A first-principles study, Phys. Rev. B 64, 115411 (2001).

[31] J. J. Palacios, A. J. Pérez-Jiménez, E. Louis, E. SanFabián, and J. A. Vergés, First-principles approach to electrical transport in atomic-scale nanostructures, Phys. Rev. B 66, 035322 (2002).

[32] E. Louis, J. A. Vergés, J. J. Palacios, A. J. Pérez-Jiménez, and E. SanFabián, Implementing the keldysh formalism into ab initio methods for the calculation of quantum transport: Application to metallic nanocontacts, Phys. Rev. B 67, 155321 (2003).

[33] J. J. Palacios et al., computer code ANT.G, publicly available at http://www.simuneatomistics.com.

[34] M. J. Frisch et al., computer code GaUSSIAN09, Revision C.01, Gaussian, Inc., Wallingford, CT, 2009.

[35] R. Dovesi et al., Crystal14: A program for the ab initio investigation of crystalline solids, Int. J. Quantum Chem. 114, 1287 (2014).

[36] K. Doll, Density functional study of Ni bulk, surfaces and the adsorbate systems $\mathrm{Ni}\left(\begin{array}{lll}1 & 1 & 1\end{array}\right)(3 \times 3) \mathrm{R} 30^{\circ}-\mathrm{Cl}$, and $\mathrm{Ni}\left(\begin{array}{lll}1 & 1 & 1\end{array}\right)(2 \times$ 2)-K, Surf. Sci. 544, 103 (2003).

[37] T. Ozaki et al., OpenMX (open source package for Material eXplorer) ver. 3.8, 2017.

[38] D. Jacob and J. J. Palacios, Orbital eigenchannel analysis for abinitio quantum transport calculations, Phys. Rev. B 73, 075429 (2006).

[39] M. R. Calvo, M. J. Caturla, D. Jacob, C. Untiedt, and J. J. Palacios, Mechanical, electrical, and magnetic properties of ni nanocontacts, IEEE T. Nanotechnol. 7, 165 (2008).

[40] N. García, M. Muñoz, and Y. W. Zhao, Ballistic magnetoresistance in transition-metal nanocontacts: The case of iron, Appl. Phys. Lett. 76, 2586 (2000).

[41] D. Jacob, J. Fernández-Rossier, and J. J. Palacios, Magnetic and orbital blocking in Ni nanocontacts, Phys. Rev. B 71, 220403 (2005). 